\title{
Costos directos y aspectos clínicos de las reacciones adversas a medicamentos en pacientes hospitalizados en el servicio de medicina interna de una institución de tercer nivel de Bogotá
}

\author{
Gabriel Tribiño, Carlos Maldonado, Omar Segura, Jorge Díaz \\ Departamento de Farmacia, Universidad Nacional de Colombia, Bogotá, D.C., Colombia.
}

Introducción. Las reacciones adversas a medicamentos constituyen un problema clínico frecuente en el ámbito hospitalario y aumentan los costos de la atención en salud. En Colombia son pocos los estudios realizados para evaluarlas desde el punto de vista clínico y económico. Objetivo. Determinar los costos generados por las reacciones adversas a medicamentos en pacientes hospitalizados en el servicio de medicina interna de una institución de tercer nivel e identificar sus principales características clínicas.

Materiales y métodos. Se hizo seguimiento intensivo de los pacientes del servicio de medicina interna durante un período de cinco meses para detectar reacciones adversas. La información se recolectó mediante un formulario basado en el formato de reporte del INVIMA. La probabilidad de causalidad se generó mediante el algoritmo de Naranjo. Se calcularon los costos directos desde la perspectiva del pagador teniendo en cuenta la estancia adicional, los medicamentos, los exámenes paraclínicos, los procedimientos, los traslados a la unidad de cuidado intermedio o intensivo y los insumos.

Resultados. Se detectaron 268 reacciones adversas en 208 de los 836 pacientes que ingresaron en el servicio (proporción de incidencia, 25,1\%; razón de presencia, 0,32). El 74,3\% se clasificó como probable; el $92,5 \%$ fue tipo A; el $81,3 \%$ correspondió a reacciones moderadas. El sistema más frecuentemente afectado fue el hematológico (33,9\%). Los medicamentos que actúan en sangre fueron los más frecuentemente relacionados $(37,6 \%)$. El costo generado por la atención de las reacciones adversas fluctuó entre \$93'633.422 y \$122'155.406.

Conclusiones. Dado el impacto negativo de las reacciones adversas en el bienestar de los pacientes, los recursos que se emplean en su atención y la proporción importante de reacciones adversas prevenibles, se requieren programas operativos de farmacovigilancia institucional.

Palabras clave: farmacoepidemiología, efectos adversos, economía de la salud, hospitalización, medicina interna

Direct costs and clinical aspects of adverse drug reactions in patients admitted to a level 3 hospital internal medicine ward.

Introduction. Adverse drug reactions (ADRs) occur frequently in hospitals and increase costs of health care; however, few studies have quantified the clinical and economic impact of ADRs in Colombia.

Objectives. These impacts were evaluated by calculating costs associated with ADRs in patients hospitalized in the internal medicine ward of a Level 3 hospital located in Bogotá, Colombia. In addition, salient clinical features of ADRs were identified and characterized.

Material and methods. Intensive follow-ups for a cohort of patients were conducted for a five month period in order to detect ADRs; different ways to classify them, according to literature, were considered as well. Information was collected using the INVIMA reporting format, and causal probability was evaluated with the Naranjo algorithm. Direct costs were calculated from the perspective of payer, based on the following costs: additional hospital stay, medications, paraclinical tests, additional procedures, patient displacement to intermediate or intensive care units, and other costs.

Results. Of 836 patients admitted to the service, 268 adverse drug reactions were detected in 208 patients (incidence proportion $25.1 \%$, occurence rate 0.32 ). About the ADRs found, $74.3 \%$ 


\begin{abstract}
were classified as probable, $92.5 \%$ were type $\mathrm{A}$, and $81.3 \%$ were moderate. The body system most often affected was the circulatory system (33.9\%). Drugs acting on the blood were most frequently those ones associated with adverse reactions (37.6\%). The costs resulting from medical care of adverse drug reactions varied from COL $\$ 93,633,422$ (USD $\$ 35,014.92$ ) to COL $\$ 122,155,406$ (USD $\$ 45,680.94$ ), according to insurance type, during the study period. Conclusions. Adverse drug reactions have a significant negative health and financial impact on patient welfare. Because of the substantial resources required for their medical care and the significant proportion of preventable adverse reactions, active programs of institutional pharmacovigilance are highly recommended.
\end{abstract}

Key words: pharmacoepidemiology, adverse effects, health economics, hospital, internal medicine

Los medicamentos han sido factor clave en la prolongación de la esperanza de vida, la erradicación o el control de ciertas enfermedades y el bienestar general de la población (1). Paralelo al desarrollo de nuevos fármacos hay un incremento en el potencial de efectos adversos; aunque los efectos terapéuticos de las nuevas moléculas pueden ser mayores y más selectivos, su seguridad debe ser evaluada continuamente (2).

Desde la década de 1960, las reacciones adversas a medicamentos se reconocen como un problema clínico frecuente y una causa importante de morbilidad (3); diversas publicaciones han mostrado que las reacciones adversas a medicamentos afectan una proporción importante de pacientes hospitalizados, y son responsables de un número considerable de hospitalizaciones y muertes cada año $(4,5)$. Además de influir negativamente en la evolución de los pacientes, las reacciones adversas a medicamentos generan un aumento significativo en los costos de los servicios de salud, principalmente en términos de estancia hospitalaria, exámenes paraclínicos, medicamentos y costos indirectos (1), por lo que la prevención de la morbimortalidad por reacciones adversas a medicamentos constituye actualmente un aspecto de importancia creciente tanto en la formación y acción de los profesionales de la salud, como en la reducción de los gastos sanitarios (6), la calidad de vida de los pacientes y la relación médico-paciente.

$\overline{\text { Correspondencia: }}$

Gabriel Tribiño E, calle $102^{\text {a }}$ \# 49-15

Teléfono: 2532154; fax 2532154

fertri@supercabletv.net.co

Recibido:31/05/05; aceptado: 18/10/05
En la literatura se menciona que los pacientes hospitalizados en los servicios de medicina interna constituyen un tercio de las admisiones, reciben un mayor número de medicamentos, presentan reacciones adversas más graves que las de otros grupos de pacientes (7-10) y su estancia hospitalaria es más prolongada $(7,11)$.

La evidencia presentada sustenta la necesidad de un sistema de farmacovigilancia. En el medio colombiano, la farmacovigilancia aún es un tema en desarrollo que involucra a los profesionales de la salud, la academia y los organismos de control y vigilancia (1).

El objetivo de este estudio fue determinar desde la perspectiva del pagador, los costos directos generados por lasreacciones adversas a medicamentos en pacientes hospitalizados en el servicio de medicina interna de una institución especializada (nivel III), estimando el monto ahorrado si se evitaran las reacciones adversas a medicamentos potencialmente prevenibles; además, identificar sus principales características clínicas, y determinar los grupos de personas y de medicamentos más frecuentemente involucrados.

\section{Materiales y métodos \\ Población de estudio}

La institución donde se llevó a cabo el estudio es un hospital universitario de tercer nivel ubicado en Bogotá, Colombia. Cuenta con 217 camas y su servicio de medicina interna consta de 42 camas; además, se encarga de la atención de un promedio de 20 pacientes ubicados en otros servicios (periféricos).

Se incluyeron todos los pacientes hospitalizados durante un período de cinco meses (noviembre 
17 de 2003 a abril 17 de 2004). Se excluyeron los pacientes hospitalizados en otros servicios sin patología relacionada con medicina interna, los pacientes para quienes la medicina interna fuera sólo especialidad de interconsulta y los pacientes hospitalizados a causa de reacciones adversas a medicamentos. Este último grupo de pacientes se excluyó puesto que presentan un contexto diferente en cuanto a tipo de medicamentos, vías de administración, cumplimiento de la terapia, seguimiento, enfermedades y entornos.

\section{Definición de caso}

Para la detección de las reacciones adversas se empleó una estrategia pasiva y una activa. La estrategia pasiva se fundamentó en una presentación inicial del proyecto y en la sensibilización de los médicos del servicio, seguida de la comunicación espontánea de los eventos adversos por parte de ellos al investigador durante el período de estudio; a partir de estos reportes, el investigador diligenció un formato de eventos adversos, modificado a partir de la forma de reporte de reacciones adversas (FORAM) del INVIMA (www.invima.gov.co). La estrategia activa implicó la revisión de las historias clínicas en busca de signos o síntomas que apuntaran hacia la presentación de un evento adverso, haciendo entrevistas o examen físico a los pacientes en caso de ser necesario. Los eventos detectados mediante esta estrategia se confirmaron con los médicos tratantes.

Se acogió la definición de reacciones adversas a medicamentos establecida por el comité técnico de la OMS en 1972 (12). Las reacciones adversas a medicamentos se caracterizaron con la terminología de reacciones adversas de la OMS (WHO-ART). Después de identificar los eventos adversos, se aplicó el algoritmo de Naranjo (2) para generar la probabilidad de causalidad; las reacciones adversas a medicamentos clasificadas como definitivas, posibles o probables se tuvieron en cuenta para el análisis; las clasificadas como dudosas fueron excluidas. En el caso de pacientes hospitalizados en medicina interna procedentes de otros servicios no se tuvieron en cuenta eventos adversos presentados en el servicio de origen; en caso de que estos pacientes presentaran eventos adversos durante su estancia en medicina interna, en el análisis se tuvieron en cuenta los medicamentos recibidos por el paciente en otros servicios.

\section{Estructura de costos}

Los costos directos generados por las reacciones adversas se calcularon teniendo en cuenta los siguientes conceptos: estancia hospitalaria adicional, medicamentos, exámenes paraclínicos, procedimientos, traslados a la unidad de cuidado intermedio o unidad de cuidado intensivo e insumos. Los costos se levantaron mediante un sistema de "microcosteo", el cual consiste en estimar cada componente de uso de recursos y derivar una unidad de costo para cada uno (13). Los costos ordenados para responder a las reacciones adversas a medicamentos se buscaron en las historias clínicas y en las hojas de facturación finales elaboradas por el departamento de cuentas médicas.

El hospital factura con base en tarifas del Servicio Obligatorio de Accidentes de Tránsito (SOAT) o del Instituto de Seguros Sociales (ISS), sometidas a ciertos ajustes según el cliente; por ello, para obtener un rango de costos se calcularon todos los conceptos (excepto medicamentos e insumos) con dichas tarifas; se emplearon las tarifas del SOAT del 2004 y del ISS del 2001; estas últimas no habían sido actualizadas por el Gobierno Nacional y, por ello, aún eran utilizadas para facturación durante el período de estudio. Las tarifas fueron suministradas por el departamento de sistemas del hospital.

Dado que el período de estudio fue menor de un año no se aplicó tasa de descuento (13).

La estancia hospitalaria adicional se calculó teniendo en cuenta el incremento por encima del promedio de hopitalización debida al diagnóstico principal del caso y ajustada según el juicio clínico del investigador.

El promedio de estancia por diagnóstico principal correspondiente al año 2003 fue suministrado por el departamento de estadística.

Para el cálculo de costos por estancia adicional se tuvieron en cuenta dos componentes: hotelería 
(que incluye habitación y servicio de enfermería) y atención diaria del paciente en medicina interna.

Para la estimación del costo anual generado por las reacciones adversas y del monto ahorrado si se evitaran las reacciones adversas prevenibles se partió de la siguiente información: a) perfil epidemiológico estable año tras año (como, de hecho, lo indicó la similitud de los perfiles revisados de los tres últimos años); b) poca diferencia en el total de pacientes atendidos anualmente en el servicio (2.224 egresos en 2001, 2.223 en 2002, 2.463 en 2003); c) promedio de estancia hospitalaria con poca variabilidad (en 2002, 8,7 días y en 2003, 8,6 días); d) razón de presencia (cociente entre el total de reacciones adversas detectadas y el total de pacientes atendidos) representativa del comportamiento anual; e) costo promedio de reacciones adversas a medicamentos calculado para los cinco meses del estudio, representativo para el resto del año, y f) proporción de reacciones adversas a medicamentos prevenibles entre 35\% y $70 \%$ según diferentes estudios publicados en la literatura $(1,2,6,9)$.

Finalmente, el costo generado por las reacciones adversas a medicamentos durante ese año se calculó teniendo en cuenta el promedio de egresos de los últimos tres años, la razón de presencia de reacciones adversas a medicamentos calculada y el costo promedio de las reacciones adversas a medicamentos calculado; el monto ahorrado se calculó considerando este valor y el rango mencionado de reacciones adversas a medicamentos prevenibles. Los resultados se obtuvieron en pesos colombianos y la conversión a dólares norteamericanos se realizó teniendo en cuenta la tasa representativa del mercado a julio 6 de 2004 (Col $\$ 2.674,1=$ US $\$ 1$ ).

\section{Análisis de datos}

Los datos recopilados se analizaron para estudiar las siguientes características de las reacciones adversas a medicamentos: medicamentos implicados, manifestaciones clínicas, órganos/ sistemas afectados, tipo, causalidad y gravedad de la reacción adversa, diagnóstico principal del caso, recursos empleados en diagnóstico y tratamiento y estancia hospitalaria adicional. Por otra parte, se determinaron diferencias entre los pacientes que presentaron reacciones adversas a medicamentos y los que no en cuanto a edad, sexo, número de medicamentos y estancia hospitalaria.

Los medicamentos implicados se definieron según la clasificación ATC (14). El tipo de reacciones adversas a medicamentos se determinó según la clasificación de Rawlins y Thopson: A, augmented (incrementada); B, bizarre (rara); E, end (terminal) (15).

El diagnóstico principal de los casos se definió según la CIE-10. La gravedad se clasificó según las definiciones de Naranjo (16), así: leve, no necesita antídoto, tratamiento ni prolongación de la hospitalización; moderada, requiere cambio en el tratamiento farmacológico, aunque no necesariamente la suspensión del medicamento causante de la reacción; grave, constituye una amenaza para la vida del paciente, requiere la suspensión del medicamento causante de la reacción y la administración de un tratamiento específico, o aquélla que causa incapacidad significativa o persistente; letal, contribuye directa o indirectamente a la muerte del paciente.

El desenlace se definió de la siguiente manera: muerte relacionada con la reacciones adversas a medicamentos; recuperación con secuelas; recuperación sin secuelas; muerte no relacionada con la reacciones adversas a medicamentos; persistencia al egreso.

La comparación entre variables cuantitativas se realizó mediante la prueba $t$ y entre variables categóricas mediante la prueba $\chi^{2}$. El nivel de confiabilidad se fijó en 0,05. Además, se desarrolló un modelo de regresión lineal escalonado para determinar el impacto de la aparición de reacciones adversas a medicamentos sobre la estancia hospitalaria, controlando el efecto de otras variables como edad, sexo, número de medicamentos y diagnósticos. La base de datos se construyó en Excel 97 y el análisis estadístico se realizó en Minitab 14.

\section{Resultados}

Durante el período de estudio ingresaron al servicio 836 pacientes con un promedio de edad de 58,9 \pm 
0,67 años, 451 (54\%) fueron hombres. La principal causa de morbilidad en la población general fue insuficiencia renal crónica; aproximadamente un tercio de los pacientes que ingresaron en el período de estudio padecían enfermedades cardiovasculares.

Se detectaron 268 reacciones adversas a medicamentos en 208 de los 836 ingresos, con una proporción de incidencia (cociente entre número de pacientes con reacciones adversas a medicamentos y total de pacientes ingresados) de $25,1 \%$ y una razón de presencia de 0,32 . Las reacciones adversas a medicamentos se presentaron en 101 hombres (48,5\%).

La media de edad de los pacientes con reacciones adversas a medicamentos fue de 59,9 919,3 años; la de los pacientes sin reacciones adversas a medicamentos fue de $58,6 \pm 19,6$ años $(p=0,39)$. A los pacientes que presentaron reacciones adversas a medicamentos se les administró en promedio $11 \pm 4$ medicamentos y a los que no, $8 \pm 3$ medicamentos $(p=0,0001)$, con lo cual se presentó un incremento de la tasa de reacciones adversas a medicamentos en aquellos que recibieron más de quince medicamentos $(60 \% \mathrm{vs}$. $21 \%, p<0,0001)$. La estancia hospitalaria para pacientes con reacciones adversas a medicamentos fue de $15,4 \pm 9,3$ días, más alta que para aquellos sin reacciones adversas a medicamentos $(8,8 \pm 6,4$ días, $p=0,0001)$.

Las categorías diagnósticas de los pacientes con reacciones adversas a medicamentos se presentan en el cuadro 1. Para las categorías diagnósticas de insuficiencia cardiaca congestiva, diabetes mellitus, angina inestable, tromboembolia pulmonar, EPOC y enfermedad por VIH no especificada, la diferencia de proporciones entre pacientes con reacciones adversas a medicamentos y sin reacciones adversas a medicamentos fue estadísticamente significativa $(p<0,05)$, siendo mayor en los primeros.

La gran mayoría de las reacciones adversas detectadas fueron de tipo A (92,5\%); $6,7 \%$ fue de tipo $\mathrm{B}$ y apenas $0,7 \%$ fue de tipo $\mathrm{E}$. Al aplicar el algoritmo de Naranjo para determinar la probabilidad de causalidad, la mayor proporción de reacciones adversas se clasificó como
Cuadro 1. Diez primeras causas de morbilidad en pacientes con reacciones adversas a medicamentos.

\begin{tabular}{lcc}
\hline Diagnóstico & Frecuencia & Porcentaje \\
\hline Insuficiencia cardiaca congestiva & 24 & 11,5 \\
Neumonía bacteriana no & & \\
especificada & 21 & 10 \\
Trombosis de vena no especificada & 18 & 8,6 \\
Evento cerebrovascular no & & \\
especificado como isquémico o & & \\
hemorrágico & 18 & 8,6 \\
Insuficiencia renal crónica & 13 & 6,2 \\
Angina inestable & 11 & 5,2 \\
Enfermedad pulmonar obstructiva & & \\
crónica con exacerbación aguda no & & \\
especificada & 11 & 5,2 \\
Diabetes mellitus no especificada & 9 & 4,3 \\
Enfermedad por VIH no especificada & 9 & 4,3 \\
Tromboembolia pulmonar & 9 & 4,3 \\
Total 10 primeras causas & $\mathbf{1 4 3}$ & $\mathbf{6 8 , 7}$ \\
\hline
\end{tabular}

Cuadro 2. Reacciones adversas más frecuentes.

\begin{tabular}{lcc}
\hline $\begin{array}{l}\text { Reacciones adversas } \\
\text { a medicamentos }\end{array}$ & Frecuencia & Porcentaje \\
\hline Exceso en anticoagulación & 78 & 29,1 \\
Hipopotasemia & 52 & 19,4 \\
Hipoglucemia & 19 & 7 \\
Bradicardia & 11 & 4,1 \\
Eosinofilia & 10 & 3,7 \\
Hemorragia de vías digestivas & 8 & 2,9 \\
Hematoma de tejidos blandos & 6 & 2,2 \\
Diarrea & 5 & 1,8 \\
Erupción cutánea & 5 & 1,8 \\
Hiperglucemia & 5 & 1,8 \\
Total & $\mathbf{1 9 9}$ & $\mathbf{7 4 , 2}$ \\
\hline
\end{tabular}

probable $(74,3 \%)$, como definitivas el $14,5 \%$ y como posibles el $11,1 \%$. En cuanto a la gravedad, la mayoría de las reacciones adversas a medicamentos se clasificaron como moderadas y leves $(81,3 \%$ y $13,4 \%$, respectivamente); el $4,1 \%$ fue grave y sólo el $1,1 \%$ resultó letal.

Las reacciones adversas a medicamentos comprendieron 49 manifestaciones diferentes, de las cuales las diez más frecuentes se exponen en el cuadro 2. Los sistemas afectados con mayor frecuencia fueron el hematológico (33,9\%), el renal $(22,7 \%)$, el gastrointestinal $(11,9 \%)$, el endocrino $(9,3 \%)$ y el cardiovascular $(8,5 \%)$. Los 
medicamentos que con más frecuencia se asociaron a reacciones adversas se presentan en el cuadro 3; los grupos anatómico terapéuticos más relacionados se presentan en el cuadro 4. Cabe anotar que la heparina utilizada con más frecuencia durante el período de estudio fue la no fraccionada, la cual se administraba mediante bomba de infusión; la fraccionada se reservaba para el tratamiento de eventos coronarios agudos.

Cuadro 3. Diez primeros medicamentos causantes de reacciones adversas a medicamentos.

\begin{tabular}{lcc}
\hline Medicamento & Frecuencia & Porcentaje \\
\hline Heparina & 81 & 30,2 \\
Furosemida & 37 & 13,8 \\
Insulina & 19 & 7 \\
Warfarina & 17 & 6,3 \\
Prednisolona & 11 & 4,1 \\
Hidrocortisona & 9 & 3,3 \\
Clonidina & 8 & 2,9 \\
Betametildigoxina & 5 & 1,8 \\
Metoprolol & 5 & 1,8 \\
Metilprednisolona & 5 & 1,8 \\
Total & $\mathbf{1 9 7}$ & $\mathbf{7 3 , 5}$ \\
\hline
\end{tabular}

Cuadro 4. Frecuencia de reacciones adversas a medicamentos por grupo de medicamento.

\begin{tabular}{lcc}
\hline Grupo ATC & Frecuencia & Porcentaje \\
\hline Sangre & 101 & 37,6 \\
Cardiovascular & 68 & 25,3 \\
Antinfecciosos & 34 & 12,6 \\
Hormonales & 27 & 10 \\
Aparato digestivo y metabolismo & 21 & 7,8 \\
Sistema nervioso & 13 & 4,8 \\
Otros & 4 & 1,4 \\
Total & $\mathbf{2 6 8}$ & $\mathbf{1 0 0}$ \\
\hline
\end{tabular}

ATC: clasificación anatómico-terapéutico-química
Cuarenta y siete reacciones adversas a medicamentos $(17,5 \%)$ generaron prolongación de la estancia hospitalaria. El promedio de estancia adicional por reacciones adversas a medicamentos fue de $4 \pm 6,9$ días (rango 1 a 46 ). Los pacientes que sufrieron reacciones adversas a medicamentos acumularon 192 días de estancia adicional; esto es el $4,2 \%$ de los días de estancia acumulados de los pacientes con reacciones adversas a medicamentos y el $1,9 \%$ de los días de estancia acumulados de la población total.

De las reacciones adversas a medicamentos detectadas, $200(74,6 \%)$ generaron costos por diferentes conceptos, discriminados de la siguiente forma: a) 124 de $268(46,2 \%)$ requirieron medicamentos como parte del tratamiento de las reacciones adversas a medicamentos; el costo por concepto de medicamentos utilizados a causa de las reacciones adversas detectadas durante los cinco meses que duró el estudio fue de \$37'218.903; b) 167 de 268 (62\%) requirieron realización de paraclínicos adicionales, el costo por este concepto osciló entre \$13'792.856 (tarifa ISS) y $\$ 22 ' 234.426$ (tarifa SOAT); c) 23 del total de reacciones adversas a medicamentos detectadas $(8,5 \%)$ requirieron la realización de diferentes procedimientos cuyo costo fluctuó entre $\$ 4^{\prime} 432.537$ (tarifa ISS) y $\$ 6^{\prime} 745.981$ (tarifa SOAT); d) en 17 de las reacciones adversas (6,3\%) se solicitaron consultas a otros servicios cuyo costo osciló entre \$1'220.060 (tarifa ISS) y \$1'867.000 (tarifa SOAT); e) 3 de las reacciones adversas $(1,1 \%)$ requirieron traslado del paciente a la unidad de cuidado intermedio; el costo por este concepto fluctuó entre \$5'970.250 (tarifa ISS) y \$7'977.500

Cuadro 5. Reacciones adversas a medicamentos que generaron los mayores costos.

\begin{tabular}{|c|c|c|c|c|c|}
\hline \multirow[t]{2}{*}{ Reacción adversa } & \multirow[t]{2}{*}{ No. } & \multicolumn{2}{|c|}{ Col \$ } & \multicolumn{2}{|c|}{ Promedio Col \$ } \\
\hline & & ISS & SOAT & ISS & SOAT \\
\hline Mielinolisis osmótica & 1 & $25^{\prime} 755.313$ & 34’315.892 & $25^{\prime} 755.313$ & $344^{\prime} 315.892$ \\
\hline HVDA & 8 & $16 ’ 552.849$ & $19^{\prime} 362.240$ & 2'069.106 & 2'420.280 \\
\hline Hipoglucemia & 19 & $15^{\prime} 400.117$ & $20 ’ 227.114$ & 810.532 & $1^{\prime} 064.584$ \\
\hline Hipopotasemia & 52 & $9^{\prime} 064.854$ & $11^{\prime} 941.354$ & 174.324 & 229.641 \\
\hline Exceso de anticoagulación & 78 & $3,180.380$ & 4'969.000 & 40.774 & 63.705 \\
\hline Insuficiencia renal & 4 & $2 ' 591.728$ & $3 ' 678.370$ & 647.932 & 919.592 \\
\hline Total 6 primeras & 162 & 72’545.241 & $94 ’ 493.970$ & NA & NA \\
\hline
\end{tabular}

HVDA: hemorragia de vías digestivas altas 
Cuadro 6. Los cinco medicamentos asociados con los mayores costos.

\begin{tabular}{lrr}
\hline Medicamento & \multicolumn{2}{c}{ Tarifa Col \$ } \\
\hline SOAT & \multicolumn{1}{c}{ ISS } \\
\hline Cloruro de sodio & $34^{\prime} 315.892$ & $25^{\prime} 755.313$ \\
Media & $34^{\prime} 315.892$ & $25^{\prime} 755.313$ \\
Insulina & $20^{\prime} 443.594$ & $15^{\prime} 570.297$ \\
Media & $1^{\prime} 075.979$ & 819.489 \\
Heparina & $19^{\prime} 802.376$ & $14^{\prime} 407.263$ \\
Media & 244.474 & 177.867 \\
Warfarina & $16^{\prime} 068.020$ & $13^{\prime} 671.615$ \\
Media & 945.178 & 804.213 \\
Prednisolona & $8^{\prime} 569.479$ & $7^{\prime} 124.296$ \\
Media & 799.044 & 647.663 \\
Total & $\mathbf{1 0 6}$ '017.795 & $\mathbf{7 6} 6^{\prime} \mathbf{5 2 8 . 7 8 4}$ \\
\hline
\end{tabular}

Cuadro 7. Costos por grupos de medicamentos relacionados con reacciones adversas a medicamentos.

\begin{tabular}{|c|c|c|c|}
\hline \multirow[t]{2}{*}{ Grupo ATC } & \multicolumn{2}{|c|}{ Tarifa Col \$ } & \multirow{2}{*}{$\begin{array}{l}\text { Porcentaj } \\
\text { del total }\end{array}$} \\
\hline & ISS & SOAT & \\
\hline angre & $53^{\prime} 836.991$ & $70^{\prime} 189.088$ & 57,5 \\
\hline Metabolismo & $15^{\prime} 665.147$ & $20 ’ 561.564$ & $16,7-16,8$ \\
\hline Hormonales & 9'054.983 & $11 ’ 082.209$ & $9,1-9,7$ \\
\hline Cardiovascular & 8'527.946 & $11^{\prime} 476.604$ & $9,1-9,4$ \\
\hline Antinfecciosos & 6’030.260 & 7’771.141 & 6,4 \\
\hline Otros & 518.095 & $1 ’ 074.800$ & $0,6-0,9$ \\
\hline Total & 93 '633.422 & $122 ' 155.406$ & 100 \\
\hline
\end{tabular}

(tarifa SOAT). Ninguna de las reacciones adversas detectadas requirió traslado del paciente a la unidad de cuidado intensivo; f) el costo por insumos empleados se ubicó entre \$14'942.331 (tarifa ISS) y \$22'942.496 (tarifa SOAT), y g) el costo por estancia hospitalaria adicional osciló entre \$16'056.485 (tarifa ISS) y \$23'169.100 (tarifa SOAT). En resumen, el costo total por la atención de las reacciones adversas a medicamentos fluctuó entre \$93'633.422 y $\$ 122 ' 155.406$. La reacciones adversas a medicamentos más costosa fue la mielinolisis osmótica. En el cuadro 5 se presentan las seis reacciones adversas que generaron mayores costos; éstas representaron el $77,4 \%$ del costo total.

El medicamento relacionado con reacciones adversas a medicamentos que más generó costos fue el cloruro de sodio; cinco medicamentos comprendieron entre el $81,7 \%$ y el $86,7 \%$ de los costos totales. Las reacciones adversas

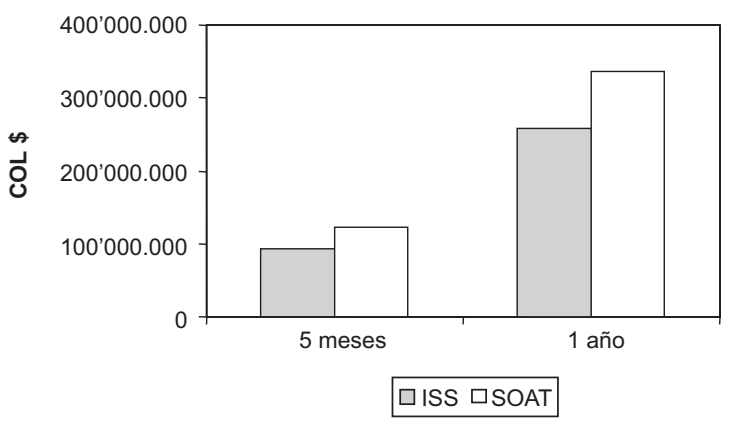

Figura 1. Proyección de costos para final del año 2004.

relacionadas con medicamentos que actúan en sangre fueron las que representaron mayores costos (cuadros 6 y 7 ).

Si se tienen en cuenta un promedio de pacientes atendidos anualmente en el servicio de 2.304 , una razón de presencia de reacciones adversas a medicamentos anual de 0,32 , un promedio de costo por reacciones adversas a medicamentos entre $\$ 349.378$ (tarifa ISS) y $\$ 455.804$ (tarifa SOAT) y un porcentaje de reacciones adversas a medicamentos prevenibles de $35 \%$, el costo al final del año fluctuaría entre \$257'841.289 (tarifa ISS) y $\$ 336$ '383.352 (tarifa SOAT, figura 1), y el ahorro al final del año si se evitaran las reacciones adversas prevenibles oscilaría entre \$90'244. 451 (tarifa ISS) y $\$ 117734.173$ (tarifa SOAT); si se acepta un porcentaje de prevenibles del $70 \%$, el monto ahorrado para el pagador se ubicaría entre $\$ 180^{\prime} 488.902$ y $\$ 235^{\prime} 468.346$.

Pasando el costo a dólares estadounidenses con base en una tasa representativa de $\$ 2.674,1$, el costo total para los cinco meses que duró el estudio osciló entre US $\$ 35.014,92$ y US $\$ 45.680,94$; el costo promedio se ubicó entre US\$130,65 y US $\$ 170,45$; el costo anual se ubicaría entre US $\$ 96.421,7$ y US $\$ 125.793,1$, y el monto ahorrado ese año fluctuaría entre US\$33.747,6 y US $\$ 44.027,6$ si se considera un $35 \%$ de reacciones adversas prevenibles, y entre US $\$ 67.495,2$ y US $\$ 88.055,2$ si se considera un $70 \%$.

El modelo de regresión lineal escalonado desarrollado reveló que el número de medicamentos y la presencia de una reacciones adversas a 
medicamentos son factores directamente asociados con el tiempo de estancia (coeficientes 0,97 y 3,07 , respectivamente; $p<0,0001$ ). De esta manera, según el modelo, la presencia de una reacciones adversas a medicamentos se asocia con un incremento en la estancia de 3 días, cifra cercana al promedio calculado mediante juicio clínico con base en el promedio de estancia por patología para la institución (4 días).

\section{Discusión}

La proporción de incidencia de reacciones adversas a medicamentos encontrada en este estudio $(25,1 \%)$ es más alta que la reportada en otras publicaciones (7,17-19), lo cual es explicable por la estrategia de vigilancia intensiva, mientras que gran parte de los estudios publicados se basan en el reporte espontáneo, cuya principal debilidad es el subreporte (7).

Por otra parte, se consideró como reacción adversa la anticoagulación excesiva, la cual aportó un número importante de casos (78 casos, $29,1 \%$ ). La anticoagulación excesiva es compatible con la definición de reacciones adversas a medicamentos de la OMS. Son pocos los estudios que incluyen la anticoagulación excesiva como reacciones adversas a medicamentos; en el estudio de Winterstein y col. (20), la reacciones adversas a medicamentos más frecuentemente reportada fue la anticoagulación excesiva, y al aplicar los criterios de Schumock y Thornton (20), ésta se constituyó en el evento adverso prevenible más frecuente.

La diferencia en el número de medicamentos administrados a pacientes con reacciones adversas a medicamentos y sin reacciones adversas a medicamentos fue estadísticamente significativa. El número de medicamentos se ha informado constantemente como un factor fuertemente asociado al desarrollo de reacciones adversas a medicamentos $(9,11,18,21,22)$.

En cuanto a la estancia hospitalaria, esta investigación encontró una diferencia estadísticamente significativa entre pacientes con reacciones adversas a medicamentos y sin reacciones adversas a medicamentos, hallazgo consistente con la literatura $(18,23)$.
La mayoría de reacciones adversas a medicamentos se clasificaron como probables según la causalidad y como moderadas según la gravedad; estos hallazgos también son compatibles con lo reportado en la literatura $(1,23,24,25)$. La mayoría de reacciones adversas detectadas fueron de tipo A $(92,5 \%)$, predominio que indica que la gran mayoría son predecibles.

A diferencia de varios estudios en los cuales los fármacos antinfecciosos son los agentes más implicados en el desarrollo de reacciones adversas a medicamentos $(9,11,19,23)$, en esta investigación los anticoagulantes fueron los más relacionados con las reacciones adversas, seguidos por los agentes que actúan a nivel cardiovascular, y los antinfecciosos ocuparon el tercer lugar; Bordet (7) ha sugerido una posible subestimación de las reacciones adversas a medicamentos inducidas por anticoagulantes.

En varios estudios $(9,11,25,26)$, las manifestaciones cutáneas fueron las más frecuentes con porcentajes entre $24 \%$ y $28 \%$; esto contrasta con los hallazgos del presente estudio, en el cual dichas manifestaciones se presentaron en apenas $2,2 \%$ de los casos; las más frecuentes fueron las que afectaron el sistema hematológico $(33,9 \%)$; en dos estudios realizados en el Hospital San Ignacio de Bogotá (19) y en el Hospital de Caldas (26), las reacciones adversas cutáneas se presentaron también con una frecuencia importante, mayor que la hallada en el presente trabajo: $28 \%$ y $20,7 \%$, respectivamente.

La alta frecuencia de reacciones adversas a medicamentos debidas a anti-coagulantes pudo obedecer a varios factores: dosificación no ajustada al peso del paciente, de manera que se administraron dosis altas; control inadecuado de tiempos de coagulación y factores relacionados con la molécula misma; a este respecto es importante señalar que según información del departamento de farmacia del hospital, durante el período de estudio se adquirió heparina de una misma marca. En cuanto a la hipopotasemia por diuréticos, la alta incidencia detectada pudo deberse a la administración de dosis altas 0 al ajuste inadecuado de la dosis según la evolución del paciente. 
La proporción de reacciones adversas a medicamentos que generó estancia adicional $(15,7 \%)$ es menor que la hallada en otro estudio, $36 \%$ (7), lo cual puede reflejar diferencias en la población considerada y los medicamentos prescritos.

Los costos generados por reacciones adversas a medicamentos presentados en la literatura (1) son más altos que los hallados por el presente estudio. Los costos calculados representan en nuestro medio entre el 6,4 y el $8,4 \%$ del PIB per cápita del país, una cifra menor que la reportada para Estados Unidos (13,4\%), dado el mayor costo de los recursos empleados en la atención en salud en dicho país.

Las reacciones adversas causadas por anticoagulantes, insulina y corticoides representaron algo más del $80 \%$ del costo global. Estos tres medicamentos causaron reacciones adversas a medicamentos con frecuencia, algunas fueron graves y generaron también el mayor número de días de estancia adicional.

Se presentaron algunas limitaciones en esta investigación. En primer lugar, es posible que la población del hospital donde se realizó el estudio presente diferencias con respecto a las poblaciones de otras instituciones, de manera que la asimilación de los resultados a otros hospitales puede ser discutible. Segundo, se reconoce que la forma más rigurosa de determinar la estancia hospitalaria adicional es mediante la diferencia entre la estancia del paciente con reacciones adversas a medicamentos y la estancia de un grupo de pacientes control apareados por variables como edad, sexo, diagnóstico y grado de gravedad. Tercero, el cálculo de los recursos ahorrados al evitar las reacciones adversas a medicamentos prevenibles usa proporciones publicadas en la literatura; no se utilizó un algoritmo para definir el carácter prevenible de las reacciones adversas a medicamentos detectadas y por ello no se calculó una proporción de reacciones adversas a medicamentos prevenibles; habría que determinar si las proporciones empleadas se ajustan a las características propias de nuestro medio. Cuarto, esta investigación incluyó sólo el servicio de medicina interna porque es aquí donde las reacciones adversas a medicamentos son más frecuentes y graves; sin embargo, dadas las diferentes patologías y medicamentos empleados en los distintos servicios, el comportamiento de las reacciones adversas a medicamentos en medicina interna puede no reflejar su comportamiento global en la institución. Por último, se incluyeron únicamente costos directos generados por las reacciones adversas a medicamentos dado que se consideró la perspectiva del pagador; sin embargo, para conocer el impacto económico global de las reacciones adversas a medicamentos es necesario determinar también los costos indirectos y los intangibles.

Los hallazgos de esta investigación confirman que las reacciones adversas a medicamentos generan un impacto negativo en la evolución clínica de los pacientes y aumentan en forma considerable los costos de la atención hospitalaria; esto, a su vez, sustenta la necesidad de establecer un programa institucional operativo de farmacovigilancia. Se ha afirmado que la implementación de estos programas en los hospitales permite la prevención o el tratamiento temprano de las reacciones adversas a medicamentos, lo cual reduce la estancia y resulta en ahorros considerables para la institución (7). El mayor ahorro logrado al evitar las reacciones adversas a medicamentos prevenibles mediante un programa de esta naturaleza se daría en términos de finanzas y disponibilidad de camas; además, mejoraría la calidad de la atención. Dado que los costos de las reacciones adversas a medicamentos en términos económicos y humanos se limitan a algunos grupos terapéuticos, los esfuerzos de prevención deben concentrarse en estos grupos para obtener una mejor relación costo-beneficio del programa (7).

\section{Conclusiones}

Las reacciones adversas más frecuentemente detectadas en los pacientes están relacionadas con las siguientes condiciones: a) edad entre los 65 y 74 años; b) tratamiento con anticoagulantes, diuréticos, corticoides e hipoglucemiantes; c) tratamiento con más de 15 medicamentos, y d) 
enfermedades cardiovasculares (insuficiencia cardíaca congestiva, trombosis venosa profunda), respiratorias (neumonía bacteriana, EPOC), insuficiencia renal crónica, diabetes mellitus y enfermedad por VIH.

La mayoría de reacciones adversas fueron tipo A, y se clasificaron como probables en cuanto a la causalidad y como moderadas en cuanto a la gravedad; por otra parte, la reacción adversa más frecuente fue la anticoagulación excesiva, y el medicamento más implicado la heparina no fraccionada. El sistema afectado con mayor frecuencia fue el hematológico.

Durante 5 meses, el costo por atención de reacciones adversas osciló entre \$93'633.422 y $\$ 122 ' 155.406$. Las reacciones adversas a medicamentos que generaron los mayores costos fueron las relacionadas con fármacos que actúan en sangre y órganos hematopoyéticos (\$53'836.991 a \$70'189. 088). Los medicamentos (\$37’218.903) y la estancia adicional (\$16’056.485 a \$23'169.100) fueron los componentes de la atención de reacciones adversas a medicamentos que generaron los mayores costos.

Cabe destacar que las reacciones adversas que más recursos consumieron eran potencialmente prevenibles: hemorragias de distinta localización por anticoagulantes, hipoglucemia por insulina, mielinólisis póntica por cloruro de sodio e hipopotasemia por diuréticos, entre otras.

Si se acepta que entre el $35 \%$ y el $70 \%$ de las reacciones adversas a medicamentos son prevenibles, el monto ahorrado en un año si se evitaran estas reacciones se ubicaría entre \$90' 244.451 y $\$ 235$ ' 468.346 .

Dado el impacto negativo de las reacciones adversas en el bienestar de los pacientes y la cantidad importante de recursos que consumen, se requiere que las instituciones hospitalarias del país implementen programas de farmacovigilancia.

\section{Conflicto de intereses}

El primer autor y los coautores del presente artículo declaramos que no teníamos conflictos de intereses de orden académico, institucional u operacional en el momento de realización de la investigación.

\section{Financiación}

Esta investigación fue realizada por el primer autor con el apoyo o tutoría de los coautores como parte de los requisitos para optar al título de Maestro en Ciencias en Farmacología de la Universidad Nacional de Colombia. Como tal, la presente investigación no contó con financiación por fuente específica (beca, subvención).

\section{Referencias}

1. Segura $\mathbf{O}$, Maldonado C. Las reacciones adversas a medicamentos: una aproximación desde el punto de vista económico. Biomédica 2003;23:401-7.

2. Bates DW, Leape L. Adverse drug reactions. En: Carruters GS et al, editors. Melmon and Morrelli's clinical pharmacology. New York: McGraw-Hill; 2000. p.1225-7.

3. Pirmohamed M, Breckenridge AM, Kitteringham NR, Park BK. Adverse drug reactions. BMJ 1998;316:12958.

4. Lazarou J, Pomeranz BH, Corey PN. Incidence of adverse drug reactions in hospitalized patients: a metaanalysis of prospective studies. JAMA 1998;279:1200-5.

5. Ajayi FO, Sun H, Perry J. Adverse drug reactions: a review of relevant factors. J Clin Pharmacol 2000:40:1093-101.

6. White TJ, Araklian A, Roh JP. Counting the costs of drug-related adverse events. Pharmacoeconomics 1999;15:445-58.

7. Bordet R, Gautier S, Le Louet H, Dupuis B, Caron J. Analysis of the direct cost of adverse drug reactions in hospitalized patients. Eur J Clin Pharmacol 2001;56:935-41.

8. Kelly WN. Can the frequency and risks of fatal adverse drug events be determined? Pharmacotherapy $2001 ; 21: 521-7$

9. Lagnaoui R, Moore N, Fach J, Longy-Bousier M, Bégaud B. Adverse drug reactions in a department of systemic disease oriented intern medicine: prevalence, incidence, costs and avoidability. Eur J Clin Pharmacol 2000;56:181-6.

10. Smith CC, Bennett PM, Pearce HM, Harrison PI, Reynolds DJ, Aronson JK et al. Adverse drug reactions in a hospital general medical unit meriting notification to Committee on Safety of Medicines. Br J Clin Pharmacol 1996;42:423-9.

11. Moore N, Lecointre D, Noblet C, Mabille M. Frequency and cost of adverse drug reactions in a department of general medicine. $\mathrm{Br} \mathrm{J}$ Clin Pharmacol 1998:45:301-8.

12. WHO. Technical Report No. 498. Diciembre 21 de 2004. Disponible en: www.who-umc.org/defs. 
13. Drummond M, O'Brien BJ, Estodart GL, Torrance GW. Análisis de costos. En: Métodos para la evaluación económica de los programas de asistencia sanitaria. Madrid: Editorial Díaz Santos; 2001. p.76-7.

14. Laporte JR, Capella D. Métodos aplicados en estudios descriptivos de utilización de medicamentos. En: Laporte JR, Tognoni G, editores. Principios de epidemiología del medicamento. Barcelona: Ediciones técnicas y científicas S.A.; 1993. p.68-9.

15. Bustamante, C. Fases del desarrollo de un nuevo fármaco. En: Ardila E, Sánchez R, Echeverry J, editores. Estrategias de investigación en medicina clínica. Bogotá: Manual Moderno; 2001. p.131.

16. Naranjo C, Busto U. Reacciones adversas a medicamentos. En: Métodos en farmacología clínica. Santiago: OPS; 1992. p.331.

17. Bates DW, Cullen DJ, Laird N, Petersen LA, Smal SD, Servi $D$ et al. Incidence of adverse drug events and potential adverse drug events. Implications for prevention. ADE Prevention Study Group. JAMA 1995;274:29-34.

18. Classen DC, Pestotnik SL, Evans RS, Lloyd JF, Burke JP. Adverse drug events in hospitalized patients. Excess length of stay, extra costs and attributable mortality. JAMA 1997;277:301-6.

19. Dennis R, Gutiérrez JM, Rodríguez MN. Creación de un programa piloto de farmacovigilancia en el Hospital Universitario San Ignacio. Acta Médica Colombiana 1998;23:15-22.
20. Winterstein AG, Hatton RC, Gonzalez-Rothi R, Johns TE, Segal R. Identifying clinically significant preventable adverse drug events through a hospital's database of adverse drug reaction reports. Am J Health Syst Pharm 2002:59:1742-9.

21. Carbonin P, Pahor M, Bernabei R, Sgadari A. Is age an independent risk factor of adverse drug reactions in hospitalized medical patients? J Am Geriatr Soc 1991; 39:1093-9

22. Nolan L, O'Malley K. Prescribing for the elderly. Part I: Sensitivity of the elderly to adverse drug reactions. J Am Geriatr Soc 1988;36:142-9.

23. Suh DC, Woodall B, Shin SK, Hermes-De Santis ER. Clinical and economic impact of adverse drug reactions in hospitalized patients. Ann Pharmacoter 2000;34:1373-9.

24. Gholami K, Shalviri G. Factors associated with preventability, predictability and severity of adverse drug reactions. Ann Pharmacother 1999;33:236-40.

25. Classen DC, Pestotnik SL, Evans RS, Burke JP. Computerized surveillance of adverse drug events in hospital patients. JAMA 1991;266:2847-51.

26. Miranda HA, Buritica OC, Ayala O. Eventos adversos a medicamentos en los servicios de medicina interna del Hospital de Caldas. Revista Médica de Risaralda 2003;9:1-7. 